\title{
¿Puede usarse radiocirugía para realizar las conizaciones diagnósticas? Estudio comparativo con la técnica tradicional con bisturí frío
}

\author{
Antonio González Mazuelo; Néstor Oswaldo Villota Cadena; Luis Guillermo Echavarría;* Luis Carlos Serna ; Juan \\ Santiago Restrepo**
}

\begin{abstract}
RESUMEN: El estudio muestra un análisis comparativo de pacientes que requerían conización diagnóstica por diversas indicaciones y que fueron realizadas con dos técnicas diferentes.

Los resultados probaron que la radiocirugía es segura para este tipo de procedimiento reduciéndose la morbilidad y los costos.

La frecuencia de compromiso en los bordes de resección, el daño térmico de los mismos y el porcentaje de lesiones finalmente no diagnosticadas fue muy semejante en ambos grupos de pacientes sin diferencias estadísticamente significativas.
\end{abstract}

Proponemos que en ciertos casos se utilice la radiocirugía para realizar las conizaciones diagnósticas.

PALABRAS CLAVES: Conización diagnóstica, radiofrecuencia, bisturí frío.

SUMMARY: The study shows a comparative analysis of patients that required diagnostic cone biopsy for diverse indications and that they were carried out with two techniques different.

The results proved that the radiosurgery is safe for this type of procedure decreasing the morbidity and the cost.

The frequency of commitment in the resection borders, the thermal damage of those same and the percentage of lesions finally not diagnosed it was very similar in both groups of patient without differences statistically significant.

We propose that in certain cases the radiosurgery be used to carry out those diagnostic cone biopsy.

KEY WORDS: Diagnostic conization, LEEP, Cold knife.

\section{Introducción}

El uso de asas diatérmicas en patología cervicouterina se propuso por primera vez en 1981 (1) formalizándose la técnica posteriormente (2) a fin de sustituir las pinzas de sacabocados en la toma de biopsias durante la colposcopia, demostrándose una mejor calidad de las muestras para el estudio histológico (3-4).

La técnica fue posteriormente adaptada al tratamiento de la neoplasia intraepitelial cervical (N.I.C) modificando la forma y las dimensiones de las asas a fines de la década de los ochenta (5) en una época dominada por los métodos destructivos locales (ablativos), principalmente la vaporización con láser de $\mathrm{CO} 2$. Estudios posteriores que compararon el procedimiento de resección con asa grande (LEEP) con láser demostraron su

Médicos Gineco-obstetras. Profesores de la Universidad Pontificia Bolivariana.

Residentes de Ginecologia y Obstetricia. Universidad Pontificia Bolivariana. igual eficacia y morbilidad (6-7) además de que, a diferencia de los métodos ablativos (crioterapia, coagulación por calor, electrocoagulación y vaporización con láser) permitía un segundo diagnóstico histológico y detectar lesiones microinvasoras ocultas no diagnosticadas durante el examen colposcópico, causantes de enfermedad invasora posterior al uso de estas técnicas.

En 1990 Shlomo Mor-Yosef (8) propuso que el LEEP podía utilizarse también como un procedimiento diagnóstico y reemplazar la conización diagnóstica tradicional hecha con bisturí frío bajo anestesia regional en las situaciones en que está indicada: colposcopia insatisfactoria o lesión parcialmente visible y/o curetaje endocervical positivo, no-correlación entre la citología y la biopsia dirigida por colposcopia y cuando esta última sugería microinvasión al estroma. Su estudio hizo una comparación con conizaciones diagnósticas cortadas con láser y demostró una frecuencia menor de complicaciones además de otras ventajas.

Una seria objeción, el daño de los bordes por el paso del asa diatérmica que impedía la apropiada lectura de 
los mismos, hizo caer en el olvido la propuesta. El uso del LEEP en el tratamiento de la N.I.C mostró en los reportes iniciales, artefactos de coagulación que interferían con la lectura histológica de los bordes de resección en el ectocervix en el $32 \%$ y en el endocervix en el $44 \%$ dificultando el diagnóstico de una excisión completa de la lesión (9).

Otra seria objeción fue la alta frecuencia inicial de compromiso de los bordes de resección ecto y endocervicales que implican excisión incompleta de la lesión y por ende la necesidad de un segundo procedimiento diagnóstico o la incertidumbre en el diagnóstico definitivo en las pacientes que iban a ser sometidas a un procedimiento terapéutico curativo posterior.

Estos hallazgos tuvieron tanto peso que un texto clásico sobre tratamiento con radiocirugía de la patología del tracto genital inferior (10) excluyó y contraindicó el uso del LEEP como equivalente de una conización diagnóstica. Solo recientemente, y producto del mejoramiento de la técnica, han vuelto a publicarse reportes sobre el uso del LEEP bajo las indicaciones en que se requiere una conización diagnóstica.

Hasta el momento, exceptuando el artículo de MorYosef, ninguno compara la técnica de LEEP con otras (láser o bisturí frío). Como en nuestro medio el uso del láser está tremendamente limitado por su costo y mantenimiento, el grupo de patología cervical de la empresa social del estado (e.s.e) Metrosalud realizó un estudio comparativo de conización diagnóstica hecha con dos técnicas: bisturí frío y LEEP a fin de evaluar las posibilidades de la segunda.

\section{Materiales y métodos}

En el período de seis años entre junio de 1991 y diciembre de 1996, 104 pacientes fueron sometidas a un procedimiento de conización diagnóstica en el centro de patología cervical y colposcopia de la e.s.e Metrosalud, institución prestadora de servicios de salud adscrita al municipio de Medellín.

El procedimiento estuvo indicado por alguna de las siguientes razones: colposcopia insatisfactoria (lesión o unión escamocolumnar parcialmente visualizadas), curetaje endocervical positivo, sospecha o confirmación de microinvasión en la biopsia dirigida, presencia de adenocarcinoma "in situ" en la biopsia dirigida y falta de correlación entre la citología y la biopsia dirigida.

Hasta diciembre de 1993 estas conizaciones fueron hechas con la técnica tradicional con bisturí frío bajo anestesia regional con la posterior hospitalización de observación por 24 a 48 horas. A partir de enero de 1994, después de la adquisición de un completo equipo de radiocirugía (LEAP 100 - Wallach - Quirurgyl) por parte de la empresa, algunas de las conizaciones diagnósticas se hicieron como un procedimiento LEEP con asa diatérmica bajo anestesia local y de forma ambulatoria con notable reducción de costos. En el período de estudio 63 pacientes se investigaron por medio de conización diagnóstica con bisturí frío y 41 pacientes con conización diagnóstica con radiocirugía.
De las indicaciones para conización diagnóstica con radiocirugía fueron excluidos los casos en que la biopsia dirigida confirma microinvasión, invasión o adenocarcinoma "in situ", circunstancias en las cuales la literatura recomienda siempre una conización con bisturi frío, bien cortado y en una sola pieza, a fin de lograr un adecuado estudio histológico. Con esta indicación se encontraron 26 conizaciones con bisturí frío y 1 conización con radiocirugía, las cuales fueron excluidas para el análisis.

37 conizaciones con bisturí frío y 40 con radiocirugía fueron finalmente seleccionadas para estudio comparativo con el fin de demostrar la equivalencia de ambos procedimientos y sustituir en el futuro la conización diagnóstica con bisturí frío por la de radiocirugía en las indicaciones seleccionadas en el presente estudio.

Estas indicaciones a su vez fueron separadas en dos grupos así :

Grupo A : incluye colposcopia insatisfactoria, curetaje endocervical positivo o sospechoso de microinvasión en la biopsia dirigida.

Grupo B : incluye únicamente la falta de correlación entre la citología y la biopsia dirigida por colposcopia.

Tanto las conizaciones con bisturí frío como las de radiocirugía fueron separadas en estos dos grupos a fin de predecir la posibilidad de que el procedimiento diagnóstico se volviera también terapéutico.

La información necesaria para el análisis comparativo fue extraída de la base de datos creada en el programa informático de la O.M.S Epi-info versión 5.0 donde se archiva la información básica de cada paciente atendida en el centro de patología cervical desde junio de 1991.

Las variables consideradas fueron analizadas comparativamente entre las conizaciones con bisturí frío y con radiocirugía. Los resultados fueron sometidos a pruebas de significancia estadística a fin de demostrar o no las diferencias con una u otra técnica.

\section{Resultados}

Durante el período analizado 1.640 pacientes fueron estudiadas en el centro de patología cervical de la E.S.E Metrosalud por anormalidades en la citología. 104 pacientes, por alguna indicación, tuvieron que someterse a un procedimiento de conización diagnóstica; esto representa una frecuencia de $6.3 \%$.

En el período de Junio de 1991 a Diciembre de 1993 se realizaron 34 conizaciones diagnósticas de 659 pacientes estudiadas en eșe lapso que representa una frecuencia de $5.16 \%$, todas realizadas con bisturí frío mientras que de Enero de 1994 a Diciembre de 1996 se realizaron 69 conizaciones diagnósticas de 981 pacientes estudiadas en ese lapso que representa una frecuencia de $7.03 \%, 28 \mathrm{con}$ técnica de bisturí frío y 41 con radiocirugía.

En general $61.2 \%$ (63 de 104) de las conizaciones se efectuaron con bisturí frío y $38.8 \%$ (41 de 104) con radiocirugía.

Excluimos del análisis, porque la biopsia dirigida reportó evidente microinvasión o adenocarcinoma, 26 conizaciones frías y una con radiocirugía. 37 conizaciones con bisturí frío y 40 con radiocirugía quedaron finalmente para el análisis comparativo. 
La edad promedio de las pacientes estudiadas con conización diagnóstica con bisturí frío (c.d.b.f) fue de 42 años (rango de 24 a 75 años) y 45 años (rango de 25 a 78 años) en las pacientes estudiadas con conización diagnostica con radiocirugía (c.d.r).

Los resultados de la citología de ambos grupos de pacientes se reportan en la tabla 1 .

Las indicaciones, divididas en subgrupos, para la conización diagnostica según la técnica se muestran en la tabla 2. No se observó diferencia importante en las indicaciones para procedimiento entre una y otra.

Solo en dos casos de c.d.r (5\%) se presentó daño térmico de los bordes de resección que impedían al patólogo dar un reporte sobre el estado de los mismos; los resultados se observan en la tabla 3 . La prueba estadística considera este porcentaje como no significativo.

Los bordes de resección resultaron comprometidos en 7 de las 37 conizaciones con bisturí frío $(\mathbf{2 4 . 3} \%)$ y en 9 de las 38 conizaciones con radiocirugía $(\mathbf{2 3 . 7} \%$ ) excluyendo de estas últimas los dos casos de bordes dañados. La prueba de signifieancia estadística no mostró diferencia. Los resultados se observan en la tabla 3.

Según la indicación para la conización diagnóstica 14 de 50 pacientes del grupo A tuvieron bordes comprometidos $(\mathbf{2 8} \%$ ) y 2 de 27 del grupo B $(\mathbf{7 . 4} \%)$. La prueba de significancia mostró diferencia importante lo cual indica que las conizaciones diagnósticas por falta de correlación cito-histológica tienen menos riesgo de borde comprometido. Los resultados se observan en la tabla 4 .

Sin embargo al comparar cada grupo por separado según la técnica utilizada no se encontró diferencia estadisticamente significativa así: en el grupo A, 7 de 25 conizaciones con bisturí frío $(28 \%)$ y 7 de 25 con radiocirugía $(28 \%)$ resultaron con bordes comprometidos y en el grupo $\mathrm{B}$, ninguna de las 12 conizaciones con bisturí frío y dos de las 15 con radiocirugía (13.3\%) resultaron con bordes comprometidos, todo lo cual indica que la posibilidad de compromiso de los bordes varía con la indicación mas no con la técnica utilizada. Los resultados se observan en la tabla 5 .

Se observó una reducción importante en el informe de bordes comprometidos en las conizaciones con radiocirugía a medida que se lograba mayor experiencia

Tabla 1

RESULTADO DE LA CITOLOGIA EN LAS PACIENTES INVESTIGADAS CON CONIZACION DIAGNOSTICA CON DOS TECNICAS DIFERENTES. METROSALUD, 1998

\begin{tabular}{|l|rr|rr|}
\hline \multirow{2}{*}{$\begin{array}{l}\text { Resultado de } \\
\text { la Citología }\end{array}$} & \multicolumn{3}{|c|}{ Técnica } \\
\cline { 2 - 5 } & \multicolumn{3}{|c|}{ Bisturí Frío } & \multicolumn{1}{|c|}{ Radiocirugía } \\
\hline Cervicitis & 0 & $0 \%$ & 0 & $0 \%$ \\
LEI de bajo grado & 1 & $2.7 \%$ & 5 & $12.5 \%$ \\
LEI de alto grado & 30 & $81.1 \%$ & 31 & $77.5 \%$ \\
Carcinoma escamoso & 6 & $16.2 \%$ & 3 & $7.5 \%$ \\
Adenocarcinoma & 0 & $0 \%$ & 1 & $0 \%$ \\
\hline
\end{tabular}

Tabla 2

INDICACION PARA LA CONIZACION DIAGNOSTICA

EN LAS PACIENTES INVESTIGADAS CON DOS TECNICAS DIFERENTES. METROSALUD, 1998

\begin{tabular}{|l|cc|cc|}
\hline \multirow{2}{*}{$\begin{array}{c}\text { Indicación } \\
\text { para el cono }\end{array}$} & \multicolumn{3}{|c|}{ Técnica } \\
\cline { 2 - 5 } & \multicolumn{2}{|c|}{ Bisturí frío } & \multicolumn{2}{|c|}{ Radiocirugía } \\
\hline Grupo A & 25 & $67.6 \%$ & 25 & $62.5 \%$ \\
Grupo B & 12 & $\mathbf{3 2 . 4} \%$ & 15 & $\mathbf{3 7 . 5} \%$ \\
Total & 37 & $100 \%$ & 40 & $100 \%$ \\
\hline
\end{tabular}

Tabla 3

DAÑO EN LOS BORDES DE RESECCION Y

COMPROMISO DE LOS MISMOS SEGUN LA

TECNICA UTILIZADA PARA LA REALIZACION DEL CONO DIAGNOSTICO. METROSALUD, 1998

\begin{tabular}{|c|c|c|c|c|c|}
\hline \multirow{2}{*}{$\begin{array}{l}\text { Daño térmico } \\
\text { de los bordes }\end{array}$} & \multicolumn{5}{|c|}{ Técnica } \\
\hline & \multicolumn{2}{|c|}{ Bisturí frío } & \multicolumn{3}{|c|}{ Radiocirugía } \\
\hline SI & 0 & $0 \%$ & 2 & $5 \%$ & \\
\hline NO & 37 & $100 \%$ & 38 & $95 \%$ & \\
\hline \multicolumn{6}{|l|}{$\begin{array}{l}\text { Compromiso } \\
\text { de los bordes }\end{array}$} \\
\hline SI & 7 & $24.3 \%$ & 9 & $23.7 \%$ & \multirow{2}{*}{ 2] } \\
\hline NO & 30 & $75.7 \%$ & 31 & $76.3 \%$ & \\
\hline
\end{tabular}

[1] Test de Fisher $p=0.2665755$, no significativo.

[2] Chi cuadrado corrección de Yates $p=0.9156885$, no significativo.

Tabla 4

COMPROMISO DE LOS BORDES DE RESECCION EN LAS CONIZACIONES DIAGNOSTICAS SEGUN LA INDICACION PARA LA MISMA. METROSALUD, 1998

\begin{tabular}{|c|cc|cr|c|}
\hline $\begin{array}{c}\text { Compromiso } \\
\text { de los bordes }\end{array}$ & \multicolumn{3}{|c|}{ SI } & \multicolumn{2}{l|}{ NO } \\
\hline Grupo A & 14 & $\mathbf{2 8} \%$ & 36 & $72 \%$ & \\
& & & & & {$[3]$} \\
Grupo B & 2 & $\mathbf{7 . 4} \%$ & 25 & $92.6 \%$ & \\
\hline
\end{tabular}

[3] Chi cuadrado, corrección de Yates $\mathrm{P}=0.0671190$, significativo. 
Tabla 5

COMPROMISO DE LOS BORDES DE RESECCION EN LAS CONIZACIONES DIAGNOSTICAS SEGUN LA INDICACION Y LA TECNICA UTILIZADA. METROSALUD, 1998

\begin{tabular}{|l|lll|ll|ll|l|}
\hline \multicolumn{7}{|c|}{ Técnica } \\
\hline \multicolumn{7}{|c|}{$\begin{array}{l}\text { Bisturí frío } \\
\text { de bordes }\end{array}$ SI } & Radiocirugía \\
\hline Indicación para el cono & NO I & NO \\
\hline Grupo A [4] & 7 & $\mathbf{2 8} \%$ & 18 & $72 \%$ & 7 & $\mathbf{2 8} \%$ & 18 & $72 \%$ \\
\hline Grupo B [5] & 0 & $\mathbf{0} \%$ & 12 & $100 \%$ & 2 & $\mathbf{1 3 . 3} \%$ & 13 & $0 \%$ \\
\hline
\end{tabular}

[4] Chi cuadrado corrección de Yates, $p=0.7527841$, no significativo.

[5] Test de Fisher, $p=0.2991453$, no significativo.

Tabla 6

POSIBILIDAD DE TRATAMIENTO DEFINITIVO EN LAS CONIZACIONES DIAGNOSTICAS SEGUN LA INDICACION Y LA TECNICA UTILIZADA. METROSALUD, 1998

\begin{tabular}{|l|c|c|c|c|}
\cline { 2 - 5 } \multicolumn{1}{c|}{} & \multicolumn{2}{c|}{ Técnica } & \multicolumn{2}{c|}{} \\
\cline { 2 - 5 } Indicación & Bisturí frío & Radiocirugía & \multicolumn{3}{c|}{ Total } \\
\hline Grupo A & 10 & 15 & $25 / 50$ & $\mathbf{5 0 \%}$ \\
Grupo B & 11 & 12 & $23 / 27$ & $\mathbf{8 5 \%}$ \\
\hline Total & $21 / 37$ & $27 / 40$ & & \\
& $\mathbf{5 6 . 8} \%$ & $\mathbf{6 7 . 5} \%$ & \multicolumn{1}{c}{} \\
& & &
\end{tabular}

Tabla 7

TUMORES MICROINVASORES NO DIAGNOSTICADOS POR LA CONIZACION SEGUN LA TECNICA UTILIZADA. METROSALUD, 1998

\begin{tabular}{|c|c|c|c|c|c|}
\hline \multirow{2}{*}{$\begin{array}{l}\text { Microinvasores } \\
\text { no diagnosticados }\end{array}$} & \multicolumn{5}{|c|}{ Técnica } \\
\hline & \multicolumn{2}{|c|}{ Bisturí frío } & \multicolumn{3}{|c|}{ Radiocirugía } \\
\hline SI & & $18.2 \%$ & & $20 \%$ & \\
\hline $\mathrm{NO}$ & 11 & $82.2 \%$ & & $80 \%$ & \\
\hline
\end{tabular}

[6] Test de Fisher, $p=0.7053571$, no significativo. con la técnica. En el período 94-95, 5 de 19 conizaciones resultaron con bordes comprometidos $(26.3 \%)$ mientras que en el año 1996 solo 4 de 22 los tuvieron (18.2\%).

Lesiones no sospechadas en el estudio de colposcopia y biopsia dirigida fueron detectadas en la conización diagnóstica; tres pacientes de las 77 estudiadas mostraron una lesión no sospechada (3.9\%). Una paciente de las c.d.b.f mostró adenocarcinoma "in situ" y dos pacientes de c.d.r mostraron microinvasión: una de adenocarcinoma (IA1 de la FIGO) y otra de escamoso (IA2 de la FIGO).

Un porcentaje importante de estas conizaciones diagnósticas pudieron volverse terapéuticas en virtud de que sus bordes resultaron negativos; 48 de las 77 conizaciones diagnósticas se volvieron terapéuticas ( 62.3 $\%)$. Según su indicación 15 conizaciones del grupo A pudieron volverse terapéuticos $(\mathbf{5 0} \%)$ y 23 del grupo B $(\mathbf{8 5} \%)$, este último resultado en concordancia con la menor frecuencia de bordes comprometidos como se mostró anteriormente. Según la técnica, 21 de las 37 conizaciones frías $(\mathbf{5 6 . 8} \%$ ) y 27 de las 40 con radiocirugía $(67.5 \%)$ pudieron volverse terapéuticos como se ve sin diferencia importante entre ambos grupos. Los resultados se observan en la tabla 6.

Tres pacientes de radiocirugía con bordes comprometidos o dudosos fueron sometidas a observación. En una de ellas se demostró persistencia de NIC III en el primer año de seguimiento y fue conizada con técnica fría. En general 30 pacientes de c.d.r fueron observadas y solo una mostró persistencia, $96.7 \%$ de curación, mientras que 21 pacientes de c.d.b.f fueron observadas y ninguna mostró persistencia, $100 \%$ de curación.

El resto de pacientes, 15 de c.d.b.f y 10 de c.d.r fueron planeadas para un segundo procedimiento con fines terapéuticos; dos pacientes se llevaron a histerectomía radical modificada y linfadenectomía por tumores microinvasores, 23 se programaron para histerectomía simple ampliada: 22 por NIC III y una por adenocarcinoma "in situ".

Sólo hubo acceso al resultado de patología después de la histerectomía simple en 16 casos, 11 de c.d.b.f y 5 de c.d.r. Se presentaron casos de tumores microinvasores no diagnosticados en la conización: dos de las 11 histerectomías por c.d.b.f tuvieron carcinoma escamoso microinvasor IA 1 (18.2 \%) y una de las 5 histerectomías por c.d.r tuvo adenocarcinoma microinvasor IA $1(\mathbf{2 0} \%)$. La prueba de significancia no mostró diferencia; los resultados se presentan en la tabla 7 .

Hubo una notoria reducción en los costos al introducir la técnica de radiocirugía para la realización de las conizaciones diagnósticas. Las 77 conizaciones con técnica de bisturí frío y hospitalización hubiesen costado a la tarifa S.O.A.T \$ 9'660.000. Las 40 conizaciones que se hicieron con radiocirugía disminuyó los costos a $\$$ 7’340.000 lo cual significó un ahorro del $24 \%$.

\section{Discusión}

Desde que se planteó por primera vez la posibilidad de utilizar la radiocirugía para realizar las conizaciones diagnósticas (8) muy pocos artículos han sido publicados apoyando este procedimiento $(11,12)$ por to cual no 
contamos con suficientes criterios objetivos para valorar la seguridad de la misma. De lo publicado, solo un trabajo se hizo comparando la radiocirugía con otra técnica (láser) y ninguno comparado con la tradicional conización con bisturi frío.

La radiocirugía cuenta ya con suficiente apoyo como técnica excisional en el tratamiento de la NIC demostrando sus notorias ventajas en comparación con otras técnicas pero su uso para procedimientos diagnósticos apenas comienza a considerarse.

El presente estudio mostró una frecuencia de conizaciones diagnósticas $(6.3 \%)$ que está dentro de lo reportado por la literatura desde que se introdujo la colposcopia en el estudio de las pacientes con citología anormal $(5-7 \%)$.

No se encontró diferencias en el promedio de edad, ni en los hallazgos de la citología en cada grupo según la. técnica (tabla 1 ).

Las indicaciones para la realización de la conización diagnóstica a saber: colposcopia insatisfactoria (lesión o unión escamocolumnar parcialmente visibles), curetaje endocervical positivo, sospecha de microinvasión o falta de correlación cito- histológica fue muy semejante en ambas técnicas (tabla 2). En el presente estudio $62.5 \%$ de c.d.r pertenecian al grupo A y $37.5 \%$ al grupo B; en el estudio Nauman (11) las indicaciones pertenecían en un $83.3 \%$ al grupo A y $16.7 \%$ al grupo B. En el presente estudio más conizaciones diagnósticas por radiocirugía se hicieron por falta de correlación cito-histológica muy semejante al estudio de Gold (12) en el cual 34.5\% de las 29 conizaciones diagnósticas hechas con radiocirugía se indicaron por esta causa.

La frecuencia de daño térmico de los bordes de resección en el presente estudio (5\%) es muy inferior a lo reportado inicialmente en la literatura. Nauman (11) reportó recientemente $1.8 \%$. Estos hallazgos son importantes ya que comparativamente con otras técnicas, la frecuencia de artefactos de coagulación no son un obstáculo para el uso de la radiocirugía en las conizaciones diagnósticas.

El compromiso de los bordes de resección es muy importante porque cuando estos son negativos podemos definir con notoria certeza el tipo de lesión histológica más avanzada presente en el cervix de la paciente y decidir con seguridad el tratamiento definitivo, cuando quiere hacerse un segundo procedimiento. Así evitamos la aparición de lesiones no sospechadas en la histerectomía simple que pudiesen requerir de terceras intervenciones nunca deseables (p.ej: radioterapia). Adicionalmente, los bordes de resección negativos permitirían bajo ciertas circunstancias volver terapéutico el procedimiento y culminar el proceso ahorrando morbilidad y costos, preservando la fertilidad.

Los estudios muestran una correlación importante de algunos factores que inciden en la lectura y compromiso de los bordes. Nauman (11) muestra que el incremento en la severidad de los cambios en la citología y el número de fragmentos que se hagan para remover la conización incrementan la posibilidad de compromiso en los bordes. Las pacientes referidas por citología sugestiva de NIC III mostraron mayores dificultades en la lectura de los bordes $(44.3 \%)$ o mayor compromiso de los mismos (16.3\%); del mismo modo, el compromiso de los bordes fue mayor cuando el cono se resecó en 2 ó más fragmentos $(44.3 \%)$ que en uno solo $(8.7 \%)$. No se observó variación en cuanto a la edad, paridad o tamaño de la lesión. En el presente estudio no se evaluaron estos factores de riesgo.

Se obtuvo una frecuencia semejante de bordes quirúrgicos comprometidos en ambas técnicas (24.3\% vs $\mathbf{2 3 . 7}$ $\%$, tabla 3 ), lo cual nos da un primer indicio de que la radiocirugía puede reemplazar en ciertas circunstancias al cono frío diagnóstico. Un reporte de 1992 (13) que incluye 300 conizaciones diagnósticas frías mostró un 40 $\%$ de compromiso de bordes por displasia; en esta experiencia solo fue del $24.3 \%$. En conización diagnóstica por radiocirugía, Mor-Yosef (8) reportó $10 \%$ de compromiso de los bordes, más baja que con técnica láser $(20 \%)$. Nauman (11) reporta $13.3 \%$ de compromiso en los bordes con conización diagnóstica con radiocirugía. En este estudio el compromiso de los bordes en los conos diagnósticos con radiocirugía fue del $23.7 \%$ y en el año 1996 bajó a $18.2 \%$, muy semejante a lo reportado.

Un factor de riesgo importante detectado para predecir el compromiso de los bordes es la indicación de la conización tanto en una y otra técnica; las pacientes con indicaciones del grupo A fue cuatro veces mayor que las del grupo B (28 \% vs $\mathbf{7 . 4} \%$ ). En el reporte de Nauman (11) $44 \%$ del grupo A tuvieron compromiso de los bordes en comparación con el solo $15 \%$ del grupo B. Esto permite que hay más pacientes en el grupo B (falta de correlación cito-histológica) puedan lograr un procedimiento terapéutico con el cono diagnóstico sin mayores diferencias entre una y otra técnica (91.6\% y $80 \%$ ).

La frecuencia de persistencia en las pacientes tratadas con conización diagnóstica fueron muy semejantes en ambas técnicas. La frecuencia de persistencia con las c.d.r fue del $3.3 \%$; Nauman (11) refiere $6.5 \%$ en las pacientes con c.d.r con bordes libres. Reportes iniciales mostraron una persistencia del $2.6 \%$ (14).

Se ha informado por el contrario, el alto riesgo de persistencia de lesiones residuales en pacientes sometidas a conización diagnóstica con radiocirugía con bordes positivos. Félix (14) reporta hasta un $63 \%$ de NIC residual cuando los bordes del cono están comprometidos; la histerectomía ( o la reconización) es usualmente recomendada a estas pacientes. En este estudio la mayoría de las pacientes con bordes positivos se programó para histerectomía; Solo tres pacientes de c.d.r con bordes positivos o dudosos fueron observadas y el seguimiento demostró un 33\% de persistencia de la NIC.

Las pacientes sometidas a un segundo procedimiento con fines terapéuticos luego de conización diagnóstica mostraron una frecuencia semejante de lesiones no presentidas por el procedimiento diagnóstico en ambas técnicas $(\mathbf{1 8 . 2} \%$ y $\mathbf{2 0} \%$ ); Ningún caso requirió de tratamiento adicional.

Se ha observado en casos de conización diagnóstica por adenocarcinoma "in situ" y bordes comprometidos una frecuencia importante de lesión residual microinvasora e invasora por lo cual se ha recomendado una reconización diagnóstica (15). En cambio en los casos de 
NIC III y bordes comprometidos esta posibilidad es remota $(5 \%)$ y puede programarse la histerectomía sin mayores riesgos.

Sin embargo en este estudio tres lesiones microinvasoras fueron diagnosticadas finalmente en la histerectomía en pacientes con cono diagnóstico previo con reporte de NIC III y bordes comprometidos, lo cual representa una frecuencia del $18.7 \%(3 / 16)$.

\section{Conclusión}

Los resultados comparativos de este estudio con la técnica fría permite concluir que la radiocirugía es segura para la conización diagnóstica en los casos de no correlación cito-histológica, colposcopia insatisfactoria y curetaje endocervical positivo como propuso inicialmente Mor-Yosef (8).

\section{BIBLIOGRAFIA}

1. Cartier, R. Sopena, B and Cartier, I. Use of the diathermy loop in the diagnosis and treatment of lesions of the uterine cervix, Proc. Fourth word congress. (1981) [Abstract].

2. Cartier, R. Practical colposcopy, 2 nd ed. Paris. Laboratorie Cartier. 1984; 139-156.

3. Atkinson, K. Symposium on cervical neoplasia, diathermy loop excision. Colpos. Gynaecol. Laser Surg. 1984-1985; 1: 285-289.

4. Prendeville, W. Davis, R. Berry, PJ. A low voltaje diathermy loop for taking cervical biopsies : A qualitative comparison with punch biopsy forceps. Br. J. Obstet. Gynaecol. 1986; 93: 773-776.

5. Prendeville, W. Cullimore, J. Norman, S. Large loop excision of the transformation zone [Letz]. A new method of the management for women with cervical intraepitelial neoplasia. Br. J. Obstet. Gynaecol. 1989; 96: 1054-1060.

6. Gunasekera, P.C. Phipps, J.H and Lewis, B.V. Large loop excision of the transformation zone [Letz] compared to carbon dioxide laser in the treatment of CIN : a superior mode of treatment. Br. J. Obstet. Gynaecol. 1990: 97: 995-998.

7. Alvarez. R.D et al. Prospective randomized trial of Lletz versus laser ablation conizacion in patients with cervical intraepitelial neoplasia. Gynecol. Oncol. 1994; 52: 175-179.

8. Mor-Yosef, S. Lopes, A. Pearson, S. Monaghan, JM. Loop diathermy cone biopsy. Obstet. Gynaecol. 1990; 75(5): 884-886.
9. Montz, FJ. Holschnider, $\mathrm{CH}$ and Thompson, LD. Large loop excision of the transformation zone [Letz]: Effect on the pathologic interpretation of resection margins. Obstet. Gynaecol. 1993; 81(6): 976-982.

10. Wrihgt, TC. Richard, RM. Ferenczy, A. Electrosurgery for HPVrelated diseases of the lower genital tract. Arthur Vision, incorporated and Biovision, New York USA and Quebec, CANADA, 1992.

11. Nauman, R. Wendel, R .et al. Lletz is an acceptable alternative to diagnostic cold-Knife conizacion. Gynecol. Oncol. 1994; 55: 224 228 .

12. Gold, M. et al. Loop electrocautery excisional procedure: therapeutic effectiveness as an ablation and conizacion equivalent. Gynecol. Oncol. 1996; 61: 241-244.

13. Paterson-Brown, S. et al. The significance of cone biopsy resection margins. Gynecol. Oncol. 1992; 46: 182-185.

14. Felix, JC. et al. The significance of positive margins in the loop electrosurgical cone biopsy. Obstet. Gynecol. 1994; 84(6): 9961000.

15. Wolf, JK. Levenback, C. et al. Adenocarcinoma in situ of the cervix: Significance of cone biopsy margins. Obstet. Gynecol. 1996; 88: 82-86. 